\title{
Cefazolin Induced Thrombocytopenia: A Rare Presentation
}

\author{
Prakash A*, Symaco E, Dalmacion DLV, Reyes D, \\ Elgohail $M$ and Ursu S \\ Department of Medicine, Monmouth Medical Center, \\ Long Branch, NewJ ersey, USA \\ *Corresponding author: Prakash A, Department of \\ Medicine, Monmouth Medical Center, $3002^{\text {nd }}$ Avenue, \\ Long Branch, NewJ ersey 07712, USA
}

Received: April 22, 2020; Accepted: May 07, 2020; Published: May 14, 2020

\begin{abstract}
Drug-induced immune thrombocytopenia is a frequent condition among hospitalized patients and is hard to diagnose because of the ambiguity of etiologies. It can lead to severe complications. We present a case of a 35-yearold male who was recently treated for infective endocarditis, now presenting with cellulitis. He was treated with cefazolin, a first-generation cephalosporin and later on developed severe thrombocytopenia. The diagnosis was difficult since our patient had multiple conditions that could explain his thrombocytopenia. This is one of the few reported cases of cefazolin-induced thrombocytopenia where we also discuss etiology, workup, and treatment in detail.
\end{abstract}

Keywords: Drug-induced thrombocytopenia; Cefazolin; Heparin-induced thrombocytopenia

\section{Abbreviations}

DIT: Drug- Induced Thrombocytopenia; HIV: Human Immunodeficiency Virus; WBC: White Blood Cell; RBC: Red Blood Cell; BNP: Brain Natriuretic Peptide; HIT: Heparin- Induced Thrombocytopenia; CT: Computed Tomography; SRA: Serotonin Release Assay, PT: Prothrombin Time; aPT: activated Prothrombin Time; INR: International Normalized Ratio

\section{Introduction}

Drug- Induced Thrombocytopenia (DIT) is a common clinical condition which is under recognized and can lead to severe bleeding complications. The diagnosis is often delayed and confounded by other potential diagnoses like infection, heparin exposure, immunologic disorders etc. Numerous medications are known to cause a variable degree of thrombocytopenia which makes identification of the offending agent as a cause of DIT extremely challenging [1]. Timely diagnosis of DIT is crucial as it can lead to catastrophic complications and also the condition is quite reversible with good outcomes. We present a rare case of cefazolin- induced thrombocytopenia.

\section{Case Discussion}

A 35- year old male with a history of HIV and intravenous drug abuse reports with progressive and exertional shortness of breath and profound edema from a duration of a week. He was recently diagnosed with methicillin-sensitive Staphylococcus aureus infective endocarditis involving tricuspid valves and treated with cefazolin for 6 weeks which got completed a week prior to admission. The patient currently denied any other signs and symptoms related to a chest infection. Notably, our patient has not been compliant with his HIV medication, bictegravir- emtricitabine- tenofovir. At the time of admission, his vitals were normal. Physical examination revealed anasarca, crackles at both lung bases, unremarkable cardiac examination, ascites without any abdominal tenderness. He had a bilateral lower extremity pitting edema with warm and erythematous skin without any ulcer. Genitourinary examination showed swollen and tense scrotum and edematous penis, no palpable inguinal lymphadenopathy. Laboratory studies showed a total WBC count of $7.5 \times 10^{3}$ cells $/ \mathrm{mm}^{3}$, hemoglobin $9.1 \mathrm{~g} / \mathrm{dL}$, platelet count of $212 \times 10^{3}$ cells $/ \mathrm{mm}^{3}$, normal chemical metabolic panel.

The patient was admitted and managed as a case of right- sided heart failure, which was confirmed with an elevated BNP level of $440 \mathrm{ng} / \mathrm{dL}$. Transthoracic echocardiogram showed severe tricuspid regurgitation as a consequence of recent infective endocarditis involving the same valve. He received intravenous furosemide for diuresis and was planned for a bioprosthetic mosaic tricuspid valve replacement. He was also being treated for cellulitis of lower extremities, for which he was started on cefazolin. His other medications at this point included aspirin and enoxaparin for venous thromboembolism prophylaxis and bictegravir- emtricitabinetenofovir. The patient was transferred to a higher center for tricuspid valve replacement. After the procedure, our patient was transferred back to our facility. At the time of readmission, his vitals were normal and physical examination was unchanged from before except for a reduction in overall edematous state. Laboratory studies showed WBC of $6.5 \times 10^{3}$ cells $/ \mathrm{mm}^{3}$, hemoglobin of $7.9 \mathrm{~g} / \mathrm{dL}$, platelet count of $174 \mathrm{X} 10^{3}$ cells $/ \mathrm{mm}^{3}$, normal chemical profile. On the following days of his hospital admission, his platelet count started to plummet with a nadir of $19 \mathrm{X}$ cells $/ \mathrm{mm}^{3}$ on day 4 .

Differential diagnosis considered at this point included HIT, idiopathic (autoimmune) thrombocytopenia, thrombotic thrombocytopenic purpura, disseminated intravascular coagulation. Coagulation studies including PT, aPT, INR, fibrinogen, D- dimer were all normal. Her initial labs did not support hemolysis and a peripheral blood smear showed normal RBCs, WBCs, non- clumped platelets. For further workup for his severe thrombocytopenia, blood cultures were drawn to rule out a possible source of infection which returned negative. His HIV 1 viral load was $<20$ copies $/ \mathrm{mm}^{3}$ (not detected) and CD4 counts were $299.1 / \mathrm{mm}^{3}$. A transesophageal echocardiogram revealed a well- seated bioprosthetic valve with no vegetations seen. 
Table 1: Mechanisms of drug-induced immune thrombocytopenia and commonly implicated medications.

\begin{tabular}{|c|c|c|}
\hline Designation & Mechanism & Examples \\
\hline Hapten-dependent antibody & $\begin{array}{l}\text { Drug (hapten) links covalently to membrane protein and induces a drug- specific } \\
\text { immune response. }\end{array}$ & Penicillin, pipericillin? cephalosporin antibiotics? \\
\hline Drug-dependent antibody & $\begin{array}{c}\text { Drug induces antibody that binds to membrane protein only in the presence of } \\
\text { soluble drug. }\end{array}$ & $\begin{array}{l}\text { Quinine, many antibiotics, non-steroidal anti- } \\
\text { inflammatory drugs, anti- convulsants }\end{array}$ \\
\hline $\begin{array}{l}\text { Fiban-induced } \\
\text { thrombocytopenia }\end{array}$ & $\begin{array}{l}\text { Drug (ligand) reacts with membrane glycoprotein IIb/IIla and induces a } \\
\text { conformational change recognized by naturally-occurring antibody? }\end{array}$ & Epitifibatide, tirofiban \\
\hline Drug-specific antibody & $\begin{array}{l}\text { Naturally occurring or induced antibody is specific for the murine component of a } \\
\text { abciximab, a chimeric Fab fragment specific for GPIIla. }\end{array}$ & Abciximab \\
\hline Autoantibody induction & Drug induces antibody that reacts with platelets in the absence of drug. & Gold salts, L-Dopa, procainamide \\
\hline Immune complex & $\begin{array}{l}\text { Drug binds to platelet factor } 4 \text { (PF4) to produce a complex for which antibody is } \\
\text { specific. The resulting immune complex activates platelets via Fc receptors. }\end{array}$ & Heparin \\
\hline
\end{tabular}

There was a strong suspicion for Heparin- Induced Thrombocytopenia (HIT) [2]. Therefore, enoxaparin was suspended and HIT antibodies, Serotonin Release Assay (SRA) were ordered. He was placed on argatroban drip. HIT antibody returned weakly positive and later serotonin assay came back negative. His platelet remained critically low thus a decision was made to replace cefazolin with vancomycin. Five days after withdrawing cefazolin, his platelet count began to rebound recovering to 160,000 cells $/ \mathrm{mm}^{3}$ over the course of an additional five days.

\section{Case Discussion}

Thrombocytopenia is a common clinical condition frequently observed in hospitalized patients. For our patient, we excluded usual diagnoses like infection and HIT, etc., which led us to consider DIT as an explanation for observed thrombocytopenia. An extensive literature search was conducted to evaluate the prevalence of cefazolin- induced thrombocytopenia with minimal findings, only a handful of cases have been reported so far [3,4]. DITP has a complex pathogenesis and can be triggered by a wide range of medications [1]. Several different mechanisms have been proposed by which drug- induced antibody- mediated platelet destruction can happen (Table 1). Cefazolin is a first-generation cephalosporin that binds to penicillin binding proteins and inhibits bacterial wall synthesis. It is generally used to treat gram- positive infections and also for surgical prophylaxis [5]. Documented adverse effects include skin rash, Steven-Johnson syndrome, anaphylaxis, diarrhea, druginduced fever, neutropenia and thrombocytopenia, etc [6]. Suggested mechanism for cefazolin- induced thrombocytopenia is that it can act as a hapten which can't trigger immunogenic response but can induce a hapten specific immune response when attached to a larger carrier protein.

The incidence of DIT is not well known but is estimated to occur in at least 10 cases per million inpatient admissions [6]. Common clinical presentation includes petechiae, bruising, epistaxis, gingival bleeding. Occasionally, the patients can present with more severe symptoms like gastrointestinal or genitourinary bleeding, renal failure, paradoxical thrombosis as seen in HIT. The presentation typically is after a week following exposure to the drug. However, onset can be rapid if the patient has been previously exposed to the drug, suggesting that drug- induced antibodies can remain in the system for longer durations [8]. DIT should be considered as a potential diagnosis in all patients who have thrombocytopenia which can't be explained otherwise. Patients having a high-pretest probability should undergo laboratory testing to identify drugrelated antibodies and hence establish the diagnosis. Treatment is the discontinuation of offending drug which usually leads to the recovery of platelet count. However, patients with severe thrombocytopenia may require platelet transfusion and other supportive measures. It is of utmost importance that the patient should never be exposed to the drug again.

\section{Conclusion}

Our patient presented as a typical case of HIT given history of the heparin exposure and temporal correlation. With the SRA results being negative and a quick rebound of the patient's platelets after discontinuation of cefazolin, it is very likely that his thrombocytopenia was linked to the usage of cefazolin.

\section{References}

1. Aster RH, George JN. Drug-induced thrombocytopenia. In: McCrae K, editor Thrombocytopenia. New York: Marcel-Dakker Inc. 2006; 145-177.

2. Davoren A, Aster RH. Heparin-induced thrombocytopenia and thrombosis. Am J Hematol. 2006; 81: 36-44.

3. Walker AS, Causey MW, Sebesta JA. "Cefazolin-induced neutropenia and thrombocytopenia following trauma: a case report". Military Medicine. 2012; 177: 352-354.

4. Drug-Induced Thrombocytopenia following a Trans vaginal Oocyte Retrieval for In Vitro Fertilization. 2009.

5. Page CP, Bohnen JM, Fletcher JR, McManus AT, Solomkin JS, Wittmann $\mathrm{DH}$. Antimicrobial prophylaxis for surgical wounds. Guidelines for clinical care. Arch Surg. 1993; 128: 79-88.

6. Homrighausen JK, Lazow SK, Berger JR. Drug-related fever due to cephazolin: a case report. J Oral Maxillofac Surg. 1999; 57: 1141-1143.

7. van den Bemt PMLA, Meyboom RHB, Egberts ACG. "Drug-induced immune thrombocytopenia," Drug Safety. 2004; 27: 1243-1252.

8. Angela L, Gerzenshtein, Lana, Ison, Michael. Antimicrobial Drug-Induced Thrombocytopenia: A Review of the Literature. Seminars in thrombosis and hemostasis. 2012; 38. 